\title{
Radiological Features and Pathognomonic Sign of Stapes Footplate Fistula in Inner Ear Malformations
}

\author{
Original Investigation $>$ (1) Levent Sennaroğlu \\ Department of Otolaryngology, Hacettepe University Faculty of Medicine, Ankara, Turkey
}

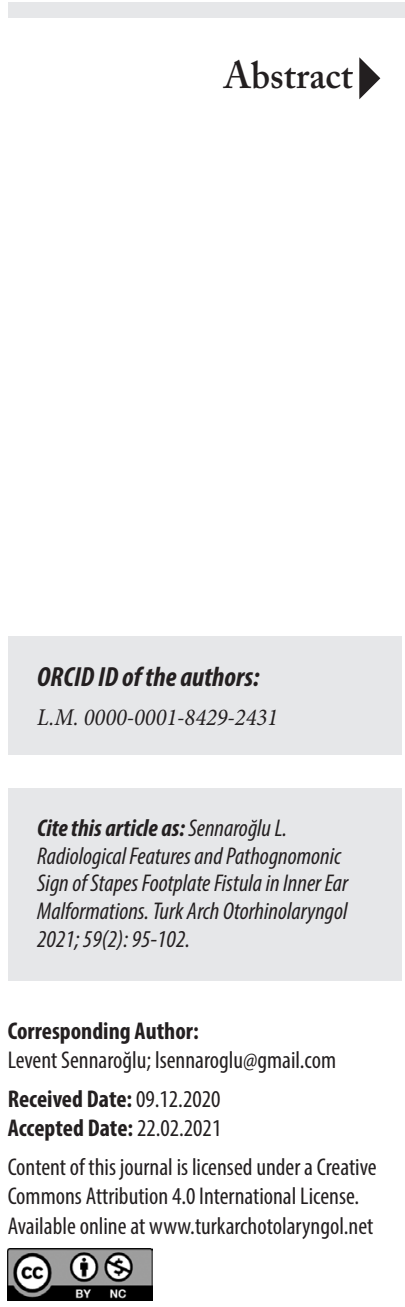

Objective: Some inner ear malformations may cause recurrent meningitis, which may be fatal. The etiology is usually a stapes footplate fistula which enables microorganisms to pass into the inner ear containing cerebrospinal fluid (CSF), causing repeated attacks of meningitis. Radiological signs of the fistula are not obvious and are not reported in detail in the literature. The aim of the study is to investigate the radiological features of stapes footplate fistula in inner ear malformations.

Methods: Radiological findings were analyzed for seventeen patients with inner ear malformations (IEMs) operated on because of recurrent meningitis. Using this information, images of 1,010 patients with IEMs were retrospectively reviewed to investigate the radiological findings of stapes footplate fistula and their relationship to IEMs. They were classified according to the Sennaroglu classification system, and according to different stages of stapes footplate fistula.

Results: In the case of a stapes footplate cyst, computerized tomography shows an opacity at the oval window. On magnetic resonance imaging, a fluid filled cystic structure continuous with and having similar signal characteristics to the CSF in the inner ear is a pathognomonic finding of a stapes footplate cyst. It is most commonly found in common cavity anomaly (18.2\%); the second most frequent finding is incomplete partition type I (15\%). And it can even be seen in cases of cochlear aplasia where only the vestibule is present.

Conclusion: If the history reveals recurrent meningitis, particular attention should be given to the oval window area, where an opacity, cyst or a leaking lesion should be looked for on the imaging. It is the responsibility of the otolaryngologist to notice these findings, and to operate on the patient to prevent further attacks of meningitis.

Keywords:Inner ear, stapes footplate fistula, congenital abnormalities, cochleovestibular anomalies, meningitis, diagnostic imaging, computerized tomography, magnetic resonance imaging 


\section{Introduction}

Meningitis is a serious disease that can be fatal. Recurrent meningitis is particularly important, because it means there is a condition that is predisposing the patient to meningitis once again (1). Inner ear malformations (IEMs) are one of the most important causes of recurrent meningitis; the disease is predominantly seen in children who have more attacks of acute otitis media when compared to adults $(1,2)$. It is the responsibility of the otolaryngologist to identify and treat the condition that causes recurring meningitis, in cases of IEMs, to prevent further complications.

In IEMs, the pathology causing recurrent meningitis is at the stapes footplate (2). Normally, the stapes footplate consists of a thin bone covered by the middle ear mucosa. During otitis media, the bony footplate provides a sufficient barrier to the medial spread of the microorganisms into the labyrinth. In the case of stapes footplate fistula, there is a bony defect at the stapes footplate, which is covered by a thin mucosal membrane or a cystic structure (2). This is not a true cyst, but a small, cerebrospinal fluid (CSF)-filled mucosal pouch in continuity with the vestibule. It is possible to have a leaking fistula as well. In the course of a middle ear infection, microorganisms pass easily through the thin membrane and defective footplate, and cause infection of the CSF immediately medial to the cyst; this results in meningitis.

Apart from being a fatal disease, in a case of single sided deafness, IEM on one side may cause meningitis and sensorineural hearing loss (SNHL) in the contralateral normal hearing side. The patient may become a candidate for a cochlear implant (CI), or even an auditory brainstem implant in cases of total ossification (Figure 1). Conditions predisposing the patient to recurrent meningitis must therefore be diagnosed and treated accordingly.

Until now, incomplete partition type I (IP-I) and cochlear hypoplasia type II (CH-II) have been regarded as the two subgroups of IEMs that may be associated with stapes footplate defect (2). Although the history may reveal recurrent meningitis and the radiologic examination demonstrates IEM, radiological signs of stapes footplate fistula are not very obvious and not well defined. Recently Mok et al. (3) published a case series showing the opacity

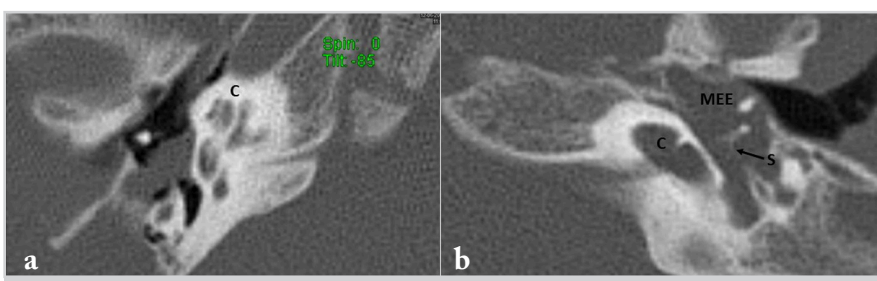

Figure 1.Cochlear hypoplasia type I (C) on the left side (a) causing meningitis and ossification on the normal hearing side on the right (b) C: Cochlea, S: Stapes, MEE: Middle ear effusion of the cyst on computerized tomography (CT); however, a study investigating all IEMs from this perspective has not yet been done. As meningitis is a serious, life-threatening condition, it is important to know which anomalies can cause meningitis, and to define its non-obvious signs.

Between 2008 and 2020, 17 cases of stapes footplate fistulas causing meningitis were operated on, and footplate defects were repaired; surgical decisions and choices of the side to be operated on were made based on imaging findings that were not very obvious. The surgical findings and outcomes of these cases are reported in a separate paper (4).

The first patient presented with a history of two attacks of meningitis after CI surgery. CT revealed fluid filling the middle ear and mastoid contralateral to the CI. Surgical indication and side selection were done according to middle ear effusion (not on the side with CI) and exploration of the middle ear revealed CSF leak and a footplate cyst. The second patient who had experienced two meningitis attacks had rounded soft-tissue density on the footplate area on the CT. This finding was thought to represent the cystic structure at the footplate, and surgery was planned accordingly. During her operation, a cyst was discovered at the footplate. From this moment on we started to look for soft-tissue density in the footplate area on CT, and for fluid (with similar intensity to CSF) filling the middle ear and the mastoid, in cases of meningitis in IEMs. More recently a cystic structure of similar characteristics, showing CSF filling the inner ear on the MRI, has also been used as an indication for middle ear exploration.

Surgical findings in these 17 patients were analyzed and reported in detail (4). The radiological findings in these patients were investigated retrospectively in 1,010 patients with IEMs; the radiological signs of stapes footplate fistula are reported in this paper.

\section{Methods}

Between 2008-2020, 17 patients who had recurrent meningitis with CSF fistula were surgically explored and the leak sites were repaired. The types of IEMs causing meningitis consisted of incomplete partition I (IP-I) and cochlear hypoplasia $(\mathrm{CH})$ cases. The decisions for surgery and the choices of the side to be operated on were made if there was an effusion filling the middle ear, soft-tissue density at the oval window on CT, or a cystic structure at the oval window area on magnetic resonance imaging (MRI) (4).

Between 1998 and 2020, the author evaluated the images of 1,010 patients with IEM. They are classified according to the eight categories of the Sennaroglu classification system, which was finalized in 2013 and published in 2017 (5). In 2020 , images of all 1,010 patients were evaluated once again in order to correlate the radiological signs of stapes footplate 
fistula with the eight categories of IEMs. Institutional approval was obtained for this study (2020/17-21).

Based on surgical and radiological data, the stapes footplate fistula is classified as follows (Figure 2)(4):

1-Normal stapes footplate (Figure 2a): A thin and intact bony stapes footplate separates the middle ear and the vestibule.

2-Stapes footplate defect (Figure 2b): There is a defect in the stapes footplate covered by the middle ear mucosa.

3-Stapes footplate cyst (Figure 2c): There is a cystic structure originating in the vestibule, passing through the stapes footplate defect into the middle ear. This can vary in size.

4-Leaking stapes footplate fistula (Figure 2d): There is a defect in the stapes footplate and CSF is leaking from the vestibule into the middle ear and the Eustachian tube.

Based on the findings from IEM cases with meningitis that were operated on, the radiological findings in stapes footplate fistula can be classified as follows (4):

1-Normal stapes footplate: The oval window area is occupied by the stapes footplate which consists of a thin bone separating the vestibule from the middle ear (Figures $3 a-b$ ).

2-Stapes footplate defect: There is a bony defect in the footplate, covered by a thin mucosal lining, and without any cystic formation (Figure 3c). Although still a histopathological finding, it may be detected by the next generation $\mathrm{CT}$ devices.

3-Opacity at the oval window on CT: A round soft-tissue density at the footplate (Figures $3 \mathrm{~d}-\mathrm{f}$ ) is an important sign of a stapes footplate cyst. It may be difficult to diagnose the opacity when there is middle ear effusion.

It is very important to differentiate the opacity at the oval window from an aberrant facial nerve and persistent stapedial artery (See, Discussion).

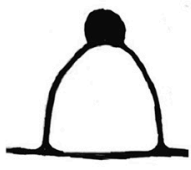

a

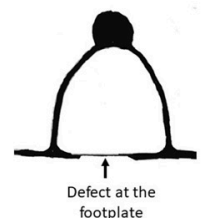

b

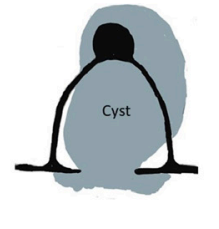

c

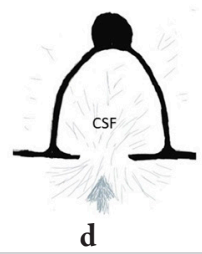

d
Figure 2. Classification of stapes footplate fistula: (a). Normal stapes footplate: Stapes has a thin and intact footplate, (b). Stapes footplate defect: Bony defect in the stapes footplate covered by middle ear mucosa, (c). Stapes footplate cyst: Cystic structure originating in the vestibule, passing through a stapes footplate defect into the middle ear, (d). Leaking stapes footplate fistula: There is a defect in the stapes footplate and CSF is leaking from the vestibule into the middle ear and from there into eustachian tube

CSF: Cerebrospinal fluid
4-Stapes footplate cyst on MRI: This is indicated if there is a fluid-filled cystic structure in the middle ear, continuous with and having similar signal intensity to the CSF in the inner ear (Figures $3 \mathrm{e}-\mathrm{g}$ ). Normally there should be no fluidfilled structure in the oval window area and the middle ear.

5-Fluid filling middle ear and mastoid: If there is fluid filling the middle ear and the mastoid, with a bright signal demonstrating similar characteristics to CSF, and fluid filling the inner ear in the CISS or FIESTA sequence on MRI (Figure 3h), this is an important sign of a leaking stapes footplate fistula. The fluid may extend via the Eustachian tube to the nasopharynx. Leaking CSF should be differentiated from fluid caused by otitis media with effusion, which has a different signal intensity on MRI (Figure 3i).

\section{Results}

The first patient with recurrent meningitis was operated on in 2008. She had bilateral IP-I anomaly and had CI surgery on the right side in 2005. On admission, she had contralateral effusion which was not present in her preoperative CT. It was therefore decided to operate on the left side. During surgery, the middle ear was filled with fluid, and there was a cyst at the footplate. Her preoperative CT demonstrated an opacity on that side that had not been noticed in 2005 at the time of her cochlear implant surgery. Therefore, as of 2008, we started to look for an opacity at the oval window area on $\mathrm{CT}$ in cases of meningitis in IEMs.

During surgery, there was a cyst at the footplate in nine children. Eight of these patients had a cystic structure on their preoperative MRI. The remaining patients had softtissue density on CT and normal findings on MRI. Therefore, if a cystic structure is present at the footplate on MRI, it can be accepted as pathognomonic for the stapes fistula cyst. Four of these patients had a CSF leak at the same time.

Twelve patients had leaking stapes fistula. One had normal $\mathrm{CT}$ and MRI, where the leak was encountered in the CI surgery. The remaining 11 patients showed fluid filling the middle ear and the mastoid on imaging. MRI was done on seven patients without prior CI surgery and demonstrated a fluid with similar signal characteristics to CSF. Three patients had prior CI surgery and underwent only CT scanning. $\mathrm{CT}$ at the time of admission demonstrated an effusion in the middle ear and the mastoid. The decision for surgery was made because of the effusion on CT, as MRI was contraindicated.

Five of the 11 patients with a leaking CSF fistula had opacity at the oval window in their original CT scanning even though there was no fluid filling the middle ear. This shows that there may be intermittent leaks from the cyst where the fluid hides the visualization of the cyst. They were all explored via an endaural approach to repair the footplate fistula. 

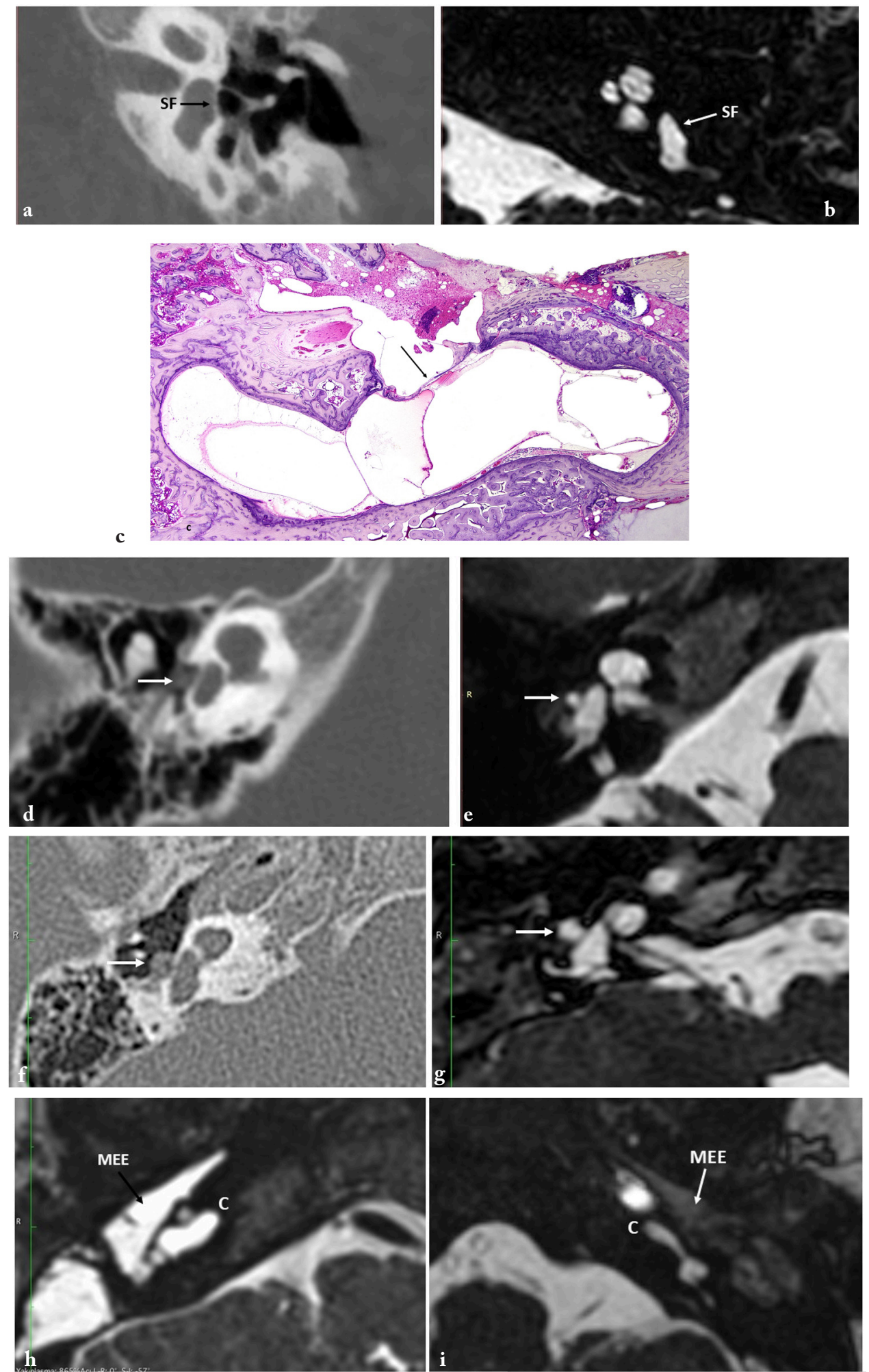

Figure 3 (a-b). Normal stapes footplate (SF) on CT (a) and MRI (b), (c). Stapes footplate bony defect covered by a thin fibrous membrane in a histopathological specimen (with Permission of the Massachusetts Eye and Ear Infirmary) (black arrow), (d-g). Computerized tomography showing a round soft-tissue density at the oval window (d-f) in IP-I cochlea. Corresponding magnetic resonance imaging demonstrates stapes footplate cyst (e-g), (h-i). Middle ear effusion (MEE). Leaking stapes fistula demonstrating MEE with a bright signal similar to cerebrospinal fluid filling the inner ear ( $h$ ). Otitis media with effusion demonstrates different signal characteristics than CSF (i)

CT: Computerized tomography, MRI: Magnetic resonance imaging 


\begin{tabular}{|c|c|c|c|c|c|c|c|}
\hline Type of IEM & $\begin{array}{l}\text { Number } \\
\text { (right and } \\
\text { left side) }\end{array}$ & $\begin{array}{l}\text { Percentage } \\
\text { within IEMs }\end{array}$ & $\begin{array}{l}\text { Middle ear effusion } \\
\text { (similar to CSF) on } \\
\text { CT or MRI }\end{array}$ & $\begin{array}{l}\text { Soft-tissue density } \\
\text { at the oval window } \\
\text { on CT }\end{array}$ & $\begin{array}{l}\text { Oval window } \\
\text { cyst on } \\
\text { MRI }\end{array}$ & $\begin{array}{l}\text { Soft tissue on CT } \\
\text { and cyst on MRI } \\
\text { present together }\end{array}$ & $\begin{array}{l}\text { No with } \\
\text { footplate } \\
\text { fistula (\%) }\end{array}$ \\
\hline IP-I & 221 & 10.9 & 7 & 28 & 29 & 23 & $34(15)$ \\
\hline IP-II & 470 & 23.3 & - & 4 & - & - & $4(0.85)$ \\
\hline $\mathrm{CH}-\mathrm{I}$ & 108 & 5.3 & - & 5 & 5 & 4 & $6(5.6)$ \\
\hline $\mathrm{CH}-\mathrm{II}$ & 114 & 5.6 & 3 & 5 & 2 & 2 & $5(4.4)$ \\
\hline CH-III & 232 & 11.5 & - & - & - & - & - \\
\hline CH-IV & 68 & 3.4 & - & - & - & - & - \\
\hline
\end{tabular}

Complete labyrinthine aplasia, rudimentary otocyst, cochlear aperture abnormalities and contralateral normal ears are excluded

IEM: Inner ear malfunction, CSF: Cerebrospinal fluid, CT: Computerized tomography, MRI: Magnetic resonance imaging, IP: Incomplete partition, EVA: Enlarged vestibular aqueduct, $\mathrm{CH}$ : Cochlear hypoplasia, $\mathrm{CC}$ : Common cavity, $\mathrm{CA}$ : Cochlear aplasia

The remaining five patients without a leaking fistula, showed rounded soft-tissue density on CT and a cyst on MRI in the oval window area. Two patients had CI surgery prior to experiencing recurrent meningitis. Both had opacity in the oval window on their original CT scanning, and this was the indication for exploration. A similar procedure was performed, with removal of the cyst and firmly packing the fistula.

In 2020 , images of 1,010 patients with IEM (2,020 ears) were retrospectively evaluated to identify the relationship of stapes footplate fistula and different groups of IEMs. There were 538 females and 472 males. The frequencies of the radiological findings are summarized in Table 1 . There were seven patients where the opacity at the stapes footplate was bilateral. Four of these cases were IP-I, and three were CC.

As can be seen in Table 1, stapes footplate fistula is most common in patients with CC (18.2\%). IP-I is second in frequency (15\%). Oval window fistula accounts for only $2 \%$ in $\mathrm{CH}$. It is encountered only in CH-I and CH-II. No patient with $\mathrm{CH}-\mathrm{III}$ and $\mathrm{CH}-\mathrm{IV}$ had a fistula at the stapes footplate. Interestingly, stapes footplate fistula can also be seen in cochlear aplasia cases: when the cochlea is absent, the round window cannot be visualized; but the oval window is present, and it can have a fistula (Figures 4a-b). It is very rare but possible in IP-II as well, but no case of IP-III had a fistula at the oval window.

Usually, the defect at the footplate is small, and there is a cyst of 2-3 mm, slightly larger than the stapes (Figure $5 \mathrm{a}$ ). In rare cases, the cyst may be very large, occupying the whole middle ear (Figure 5b).
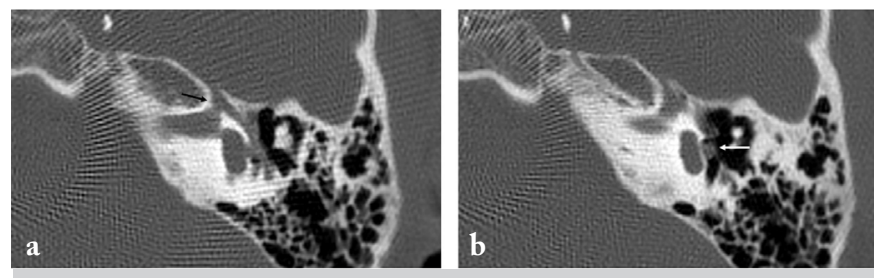

Figure 4. Cochlear aplasia with dilated vestibule (a) where labyrinthine segment of the facial nerve is at the location of the absent cochlea (white arrow). (b) Opacity is shown in the oval window area (black arrow)
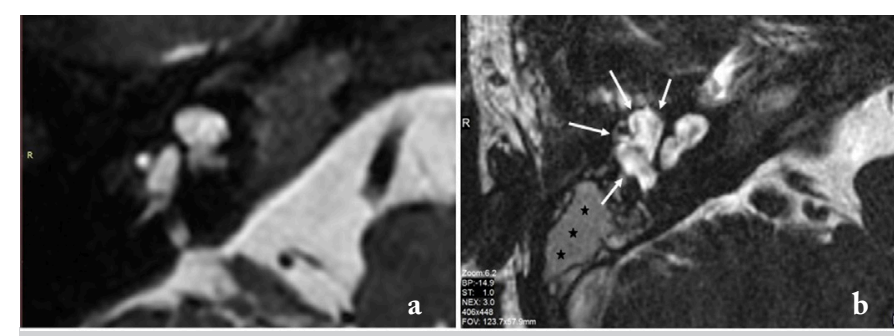

Figure 5. Different sizes of stapes footplate cyst; usually 2-3 mm cyst (a) is present at the oval window. Rarely a cyst filling the whole middle ear can be seen (b) (thin white arrows). Otitis media with effusion demonstrates different signal intensity (black stars) than CSF filling the cyst

CSF: Cerebrospinal fluid

\section{Discussion}

Recurrent meningitis in IEMs is a serious condition and has to be diagnosed and treated appropriately. It may be fatal, or cause hearing loss and ossification on the contralateral normal hearing ear in single sided deafness. Every effort must therefore be made to diagnose and treat the condition before it leads to meningitis. 
Histopathology: In IEMs, the stapes footplate may have a defect which is covered by a thin membrane (Figure 3c). This defect has been reported mainly in IP-I and rarely in $\mathrm{CH}$ and $\mathrm{CC}$ (5-10). According to the database of Hacettepe University's Department of Otolaryngology, IP-I was found in $10.9 \%$ and $\mathrm{CC}$ in $5.9 \%$ of 1,010 patients with IEM. Therefore, $\mathrm{CC}$ is much rarer, and the histopathology of a true case of $\mathrm{CC}$ has not been reported. The presented study shows that the percentage of stapes footplate fistula is highest in the CC group. IP-I, CH-I, CH-II and CC are all endosteal developmental malformations (2). Therefore, a footplate defect is possible in all these cases.

One of the most interesting findings is the presence of a fistula in cochlear aplasia. When the cochlea is absent, the round window is absent, but the oval window is present. Three cases (5\% of cochlear aplasia cases) demonstrated findings of stapes footplate fistula. All of these were cases of cochlear aplasia with dilated vestibule. When the vestibule is dilated, it is possible to think of an endosteal developmental abnormality. This supports the above explanation.

Pathophysiology: Normally, the oval window is occupied by the stapes footplate, which is a thin, bony structure with middle ear mucosa on top; it prevents the spread of middle ear infection into the inner ear and CSF-containing area. But if there is a stapes footplate defect or a cyst, this creates a defective area. In a case of acute otitis media, microorganisms can pass easily through the thin membrane into the CSFcontaining area, causing meningitis. This condition is mainly seen in children who are more prone to acute otitis media than adults (2). If there is no middle ear infection, this defect may not be noticed at all throughout the life of the patient. Our patients with stapes footplate fistula had meningitis in spite of their having had a pneumococcus vaccination (4). Therefore, while vaccination is very important in patients with IEMs, it may not prevent meningitis in cases of stapes footplate cyst. None of the patients had meningitis after footplate repair; it is therefore important to diagnose and repair the footplate defect, even if it is asymptomatic.

Until 2014, high CSF pressure was held responsible for causing the stapes footplate defect in IP-I (1). Evaluation of IEMs in the Massachusetts Eye and Ear Infirmary revealed that it was possible to have a footplate defect even in the presence of a thin but intact modiolar base (Figure 3c) (2). This shows that it is not the CSF pressure causing the defect. Sennaroglu (2) explained this finding in terms of an endosteal developmental anomaly, which is the result of decreased blood flow from the IAC. This results in a very thin and defective endosteum (the innermost layer of the otic capsule) all around the cochleovestibular space, while the middle enchondral and outer periosteal layers are normal. The same explanation holds for CC, CH-I, and CH-II.
In IP-III there is a large defect at the base of the cochlea. These cases had a $100 \%$ risk of a CSF gusher during CI surgery, but none had a footplate fistula and recurrent meningitis. There is no histopathological specimen with IPIII, but it can be interpreted from the temporal CT scanning that there is a thick endosteal layer in IP-III, while the outer two layers of the otic capsule are missing. This causes stapes fixation without any fistula at the stapes footplate. Therefore, spontaneous stapes footplate fistula formation in IP-III has not been observed by our group or reported in the literature. This also highlights the fact that high CSF pressure cannot be held responsible for spontaneous stapes footplate fistulas.

The defect at the footplate is present at birth, but if there is no acute otitis media, the defect is not noticed, and patients live with it for the rest of their lives. In the event of a middle ear infection, it causes meningitis. If there is a bony separation between the IAC and the cochlea (preventing entry of CSF into the inner ear), the footplate defect may not be noticed at all during the patient's lifetime. A footplate fistula can only be noticed if there is a middle ear infection, which may cause meningitis when the infection passes through the thin membrane at the footplate, and into the CSF filling the cochlea.

We have seen patients with stapes footplate fistula who had meningitis despite having had pneumococcus vaccination. After the infection, the middle ear should be explored, and the fistula repaired. In all the cases that we explored the fistula was at the oval window. Once the fistula is repaired by a millimetric piece of fascia inserted into the vestibule in a dumbbell fashion, this provides a sufficient barrier, preventing the spread of microorganisms into CSF-containing areas, hence preventing meningitis.

Terminology: There are different terms used to describe this situation. As the pathology is at the stapes footplate, the best terminology appears to be stapes footplate fistula. A fistula is an abnormal connection between two spaces. Because it can happen even in cases of cochlear aplasia, "cochlear fistula" may not be an appropriate term. If there is continuous leakage, the term "leaking stapes footplate fistula" describes the situation correctly. On CT, we cannot differentiate the soft-tissue density, so the best terminology appears to be "round soft-tissue density at the stapes footplate." On MRI, if we observe a fluid-filled structure continuous with inner-ear fluid and CSF, it is termed "stapes footplate cyst." During surgery, the author removed the cystic structure from the footplate defect. However, a real cyst does not have any opening into any other surface, and therefore this pathology is different from a true cyst. Likewise, the term "cochleocelle" used by Mok et al. (3) may not be appropriate because the pathology does not originate from cochlea, and it can be observed even in the absence of a cochlea. One suggestion 

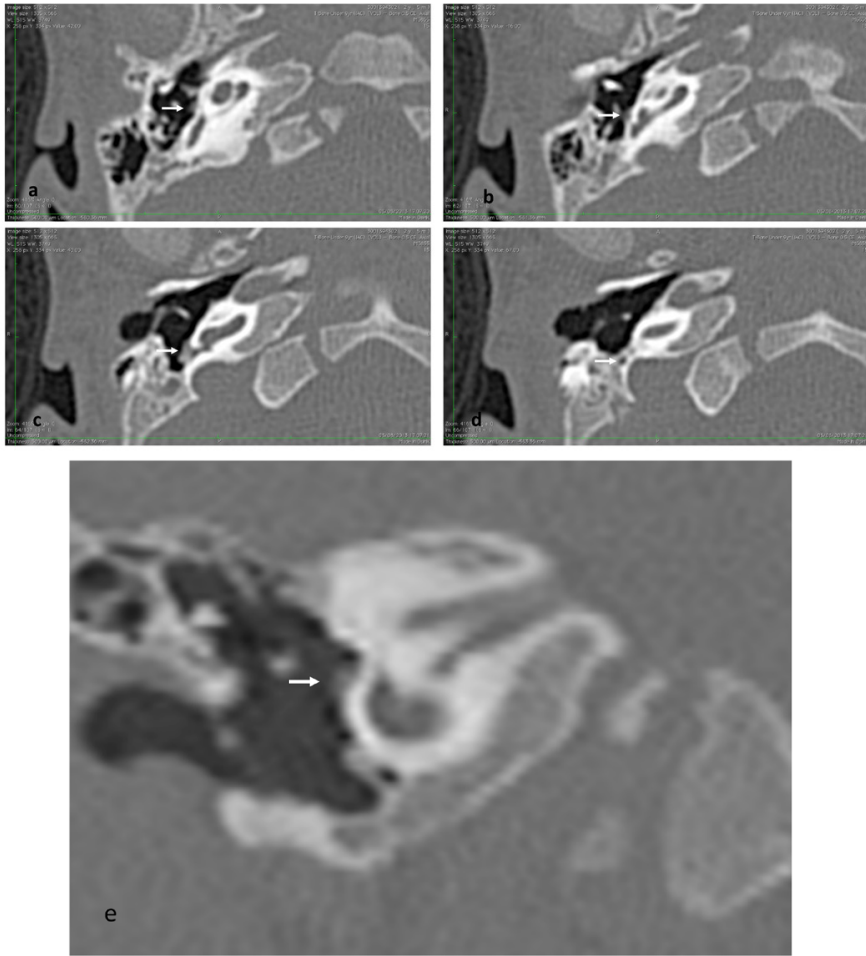

Figure 6. Aberrant facial nerve. Computerized tomography showing soft-tissue density (white arrow) around the oval window area (b) which can be followed proximally to the labyrinthine segment and distally to the mastoid segment on axial sections $(\mathrm{a}-\mathrm{d})$. On coronal section facial nerve (white arrow) is located inferior to oval window (e)
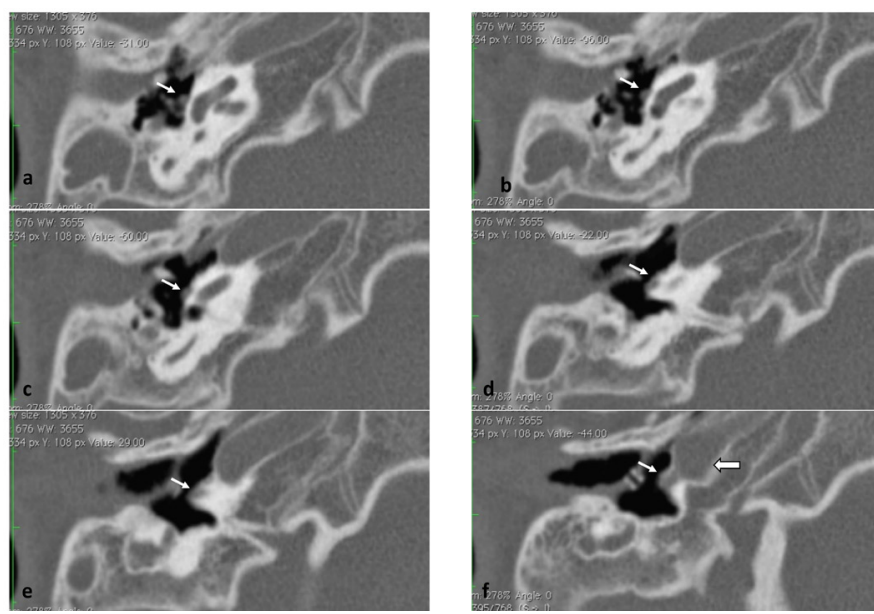

Figure 7. Persistent stapedial artery. Computerized tomography (a) shows a soft-tissue density in the left oval window area (thin white arrow). When followed distally it is located on the promontory and opens into carotid artery (thick white arrow) (a-f)

is to use the term "endosteocele;" this may be appropriate if we think of the origin of the cystic structure as the inner ear endosteum.

Imaging: Why is the sign on MRI pathognomonic? If we see a cystic structure in the oval window area in the middle ear, continuous with the vestibule and having bright signal on high-resolution T2 weighted images such as CISS or FIESTA sequences, this indicates that it is coming from the inner ear. If the fluid signal is widespread, it may be a leaking fistula. The soft-tissue density on the CT must be differentiated from an aberrant facial nerve and persistent stapedial artery.

It is very important that the otolaryngologist summarizes the patient's history in the request for imaging, particularly mentioning meningitis. If there is a history of recurrent meningitis, the stapes footplate area should be carefully evaluated on CT and MRI. If soft-tissue density or a cyst is noticed on imaging, it must be highlighted in the report. A previous study demonstrated that a slice-thickness of 0.5 $\mathrm{mm}$ for CT and MRI is appropriate to demonstrate the findings of stapes footplate cyst (4).

\section{Radiological Signs of Stapes Footplate Fistula} Necessitating Surgery: None of the patients who were operated on had a clear diagnosis of fistula before exploration. In cases of recurrent meningitis, the presence of the following points may be a sign of stapes footplate fistula necessitating middle ear exploration:

1. IEM of the subgroup: Common Cavity, IP-I, CH-I, CHII, Cochlear aplasia

2. A round soft-tissue density at the oval window area on CT

3. Cystic structure in the oval window area on MRI

4.Middle ear effusion in the above IEM types, continuous and having a similar signal to the CSF-containing inner ear on MRI, which may extend through the Eustachian tube into the nasopharynx

As surgeons, we should not forget that if there is a suspicion of a CSF leak it is advisable to go ahead with surgery rather than to wait and see.

Special attention is necessary in patients with common cavity. In some of these cases, the vestibule is hypoplastic, and if the area is not evaluated with thin-section, high-resolution CT imaging, footplate cyst may not be demonstrated.

Differential Diagnosis: It is important to differentiate this condition from other pathologies at the oval window.

1. Aberrant facial nerve at the oval window. This has a characteristic finding on coronal CT (Figure 6e). There is no neural tissue at the usual location of the facial nerve, which is normally located inferior to lateral SCC, superior and lateral to oval window. When the soft tissue is followed proximally and distally on axial sections (Figures 6a-d) the canal for the facial nerve can be differentiated easily from the cyst which is located only at the oval window. In stapes footplate fistula, 
facial nerve is at its usual location. In cases of aberrant facial nerve, there is no fluid signal indicating a cystic structure on MRI.

2. Persistent stapedial artery. There is a rounded soft-tissue density at the oval window. It can be followed distally until it is connected to the internal carotid artery (Figures 7af). If there is a stapes footplate cyst it cannot be followed proximally and distally as can be in the latter two situations.

\section{Conclusion}

In inner ear malformations, meningitis may be fatal but may also cause SNHL on the contralateral normal hearing side in single-sided deafness. Patients with single-sided deafness therefore need to undergo CT and MRI, for the purpose of carefully evaluating the footplate, and not missing an oval window fistula that may result in meningitis. It is very important to diagnose a stapes footplate fistula preoperatively. A cystic structure on MRI is pathognomonic, but a round soft-tissue density on CT should arouse suspicion: these are indications for middle ear exploration. Stapes footplate fistula is most common in common cavity and incomplete partition type I cases.

Ethics Committee Approval: Institutional approval was obtained for this study (Hacettepe University Faculty of Medicine, 2020/17-21).

\section{Informed Consent: Retrospective study.}

Financial Disclosure: The author declared that this study has received no financial support.

Peer-review: Externally peer-reviewed.

\section{References}

1. Sennaroglu L. Cochlear implantation in inner ear malformations-a review article. Cochlear Implants Int 2010; 11: 4-41. [Crossref]

2. Sennaroglu L. Histopathology of inner ear malformations: Do we have enough evidence to explain pathophysiology? Cochlear Implants Int 2016; 17: 3-20. [Crossref]

3. Mok F, Leonard CG, Adams MA, Trimble K, Papsin BC, James AL, et al. Total endoscopic ear surgery in management of cochleocele: A case series. Int J Pediatr Otorhinolaryngol 2020; 135: 110109. [Crossref]

4. Sennaroglu L, Bajin MD. Management of stapes footplate fistula in inner ear malformations. Int J Pediatr Otorhinolaryngol 2020; 140: 110525. [Crossref]

5. Sennaroglu L, Bajin MD. Classification and current management of inner ear malformations. Balkan Med J 2017; 34: 397-411. [Crossref]

6. Phelps PD, King A, Michaels L. Cochlear dysplasia and meningitis. Am J Otol 1994; 15: 551-7. [Crossref]

7. Syal R, Tyagi I, Goyal A. Cerebrospinal fluid otorhinorrhea due to cochlear dysplasias. Int J Pediatr Otorhinolaryngol 2005; 69: 983-8. [Crossref]

8. Zwierz A, Masna K, Burduk P. Recurrent meningitis in congenital inner ear malformation. Ear Nose Throat J 2020:145561320920399. [Crossref]

9. Wani NA, Rawa A, Qureshi U, Kosar T, Robbani I. Recurrent meningitis in an adult secondary to an inner ear malformation: imaging demonstration. Ear Nose Throat J 2012; 91: E23-6. [Crossref]

10. Ramesh V, Sankar J, Krishnamurthy B. Recurrent pneumococcal meningitis in a child with congenital common cavity malformation of the inner ear. Clin Pediatr (Phila) 2014; 53: 397-8. [Crossref] 